\title{
ISUP Grade Pattern 1
}

National Cancer Institute

\section{Source}

National Cancer Institute. ISUP Grade Pattern 1. NCI Thesaurus. Code C162654.

Circumscribed nodule of closely packed but separate, uniform, rounded to oval, mediumsized acini (larger glands than pattern 3). 